\title{
The expenditures related to the use of antifungal drugs in patients with hematological cancers: a cost analysis
}

This article was published in the following Dove Press journal:

ClinicoEconomics and Outcomes Research

3 November 2015

Number of times this article has been viewed

\section{Habip Gedik}

Department of Infectious Diseases and Clinical Microbiology, Ministry of Health Okmeydanı Training and Research Hospital, Istanbul, Turkey
Correspondence: Habip Gedik Bakırköy Dr.Sadi Konuk Eğitim ve Araștırma Hastanesi, Zuhuratbaba Mh., Tevfik Sağlam Cad. No: I I, Bakırköy/ İstanbul, Turkey

Tel +90 5053362770

Email habipgedik@yahoo.com
Objective: The aim of this study is to analyze the expenditures related to the use of antifungal drugs in patients with hematological malignancies.

Methods: In this retrospective study, the expenditures related to use of antifungal drugs for treatment of invasive fungal infections in patients with hematological malignancies between November 2010 and November 2012 were analyzed. Expenditures of antifungal drugs were calculated by converting the price billed to the Republic of Turkey Social Security Institution per patient using the US dollar (\$) exchange rate.

Results: We retrospectively analyzed the expenditures related to the use of antifungal drugs in 282 febrile episodes of 126 neutropenic patients. Voriconazole (VOR), caspofungin, and liposomal amphotericin $\mathrm{B}(\mathrm{L}-\mathrm{AmB})$ were administered as a first-line antifungal therapy to treat 72 febrile episodes of 65 neutropenic patients, 45 febrile episodes of 37 neutropenic patients, and 34 febrile episodes of 32 neutropenic patients, respectively. The expenditures related to the use of antifungal drugs per febrile neutropenic episode were $\$ 3,857.85$ for VOR; $\$ 15,783.34$ for caspofungin, and $\$ 21,561.02$ for L-AmB, respectively. The expenditure related to the use of posaconazole (POS) was $\$ 32,167.39$ per patient for primary or secondary prophylaxis.

Conclusion: Improving conditions in the patient's room, choosing pre-emptive antifungal treatment instead of empirical antifungal treatment, switching to tablet form of VOR after initiation of its intravenous form, secondary prophylaxis with VOR against invasive aspergillosis, primary prophylaxis with POS in high-risk patients, and choosing less L-AmB as being an alternative to other antifungal drugs, may reduce expenditures related to the use of antifungal drugs in the treatment of invasive fungal infections during febrile neutropenic episodes of patients with hematological malignancies. Keywords: hematological malignancies, invasive fungal infection, expenditure, antifungal drug, neutropenia

\section{Introduction}

Febrile neutropenia $(\mathrm{FN})$ is mainly a complication of cancer chemotherapy and defined as an oral temperature of $>38.3^{\circ} \mathrm{C}$ or two consecutive measurements of $>38.0^{\circ} \mathrm{C}$ over 2 hours and also an absolute neutrophil count of $<0.5 \times 10^{9} / \mathrm{L}$ or expected to decrease below $0.5 \times 10^{9} / \mathrm{L}^{1-3}$ Bacteremia caused by Gram-positive and -negative bacteremia and invasive fungal infections (IFIs) caused by Aspergillus spp., Candida spp., Zygomycetes, etc are commonly cause mortality, in addition to hematological malignancy-related complications. Antibacterial and antifungal drugs have important roles in the treatment of febrile neutropenic episodes. Treatment is initiated based on empirical antibiotic therapy subsequent to the appearance of fever during a neutropenic episode, followed by microbiological and radiological results. ${ }^{1-3}$ However, a definite diagnosis of invasive infection versus colonization is often difficult. Chemotherapy for hematological cancers, 
the growing incidence of fungal infections associated with neutropenia and consequently growing expenditures related to their treatment have been increasing worldwide. Especially, antifungal drugs cost more than antibacterial drugs. The systemic antifungal market increased in value from $\$ 2.1$ billion in 1999 to $\$ 3.3$ billion in 2003. The azole antifungal drugs have dominated the systemic antifungal market, accounting for $52 \%$ of total sales in $2003 .{ }^{4}$ Previous pharmacoeconomic studies compared either voriconazole (VOR) or caspofungin (CAS) with liposomal amphotericin B (L-AmB) that were used as empirical therapy for treating FN episodes..$^{5-7}$ In some health care settings, the incidence of IFIs has been reported to increase with more intense use of antifungal drugs. ${ }^{3}$

In this retrospective study, we aimed to analyze the expenditures related to the use of antifungal drugs in patients with hematological malignancies.

\section{Materials and methods}

In this retrospective study, the expenditures related to the use of antifungal drugs in the treatment of IFIs during febrile neutropenic episodes of patients who were aged over 14 years and received chemotherapy for hematological malignancies between November 2010 and November 2012 were analyzed. The ethics committee of the Health Okmeydanı Training and Research Hospital, which is an 800-bed tertiary hospital in İstanbul, Turkey, approved this study. Patients were evaluated if they had experienced at least one neutropenic episode due to hematological cancer chemotherapy in the hematology ward. Patients treated for other hematological diseases (eg, anemia, idiopathic or immune thrombocytopenic purpura, etc) were not evaluated. The hematology department was equipped with 23 beds in single, double, and quadruple rooms without high-efficiency particulate air filters. In the hematology ward, patients and their attendants were residing in the same room and sharing three toilets. A nurse and a doctor of the hematology ward were performing a weekly 1-hour instructional program regarding drug-resistant microorganisms and preventative measures to patients and their attendants. FN was defined as an oral temperature of $>38.3^{\circ} \mathrm{C}$ or two consecutive measurements of $>38.0^{\circ} \mathrm{C}$ over 2 hours, and also an absolute neutrophil count of $<0.5 \times 10^{9} / \mathrm{L}$ or expected to decrease below $0.5 \times 10^{9} / \mathrm{L}{ }^{1}$ The patients' demographics and diagnosis, neutropenic episodes, clinical presentations and laboratory findings, antimicrobial treatments, and microbiological data and outcomes were evaluated. The management of FN was based on the clinical practice guidelines of both the Infectious Diseases Society of America and the European Organization for Research and Treatment of Cancer/Invasive Fungal Infections Cooperative Group and the National Institute of Allergy and Infectious Diseases
Mycoses Study Group. ${ }^{1-3}$ The scoring system was being used to score the risk of complications in patients with FN episodes based on the Multinational Association for Supportive Care in Cancer score. ${ }^{1}$

Empirical antifungal treatment was being administered for patients with persistent or recurrent fever after 4-7 days of antibiotics and whose overall duration of neutropenia was being expected to be more than 7 days. ${ }^{1-3}$ The preemptive treatment strategy was defined as follows: mold-active antifungal drugs were started when computed tomography (CT) showed infiltrate or nodule(s) and/or serum galactomannan became positive in patients with $\mathrm{FN}$ in this study. If CT showed changes associated with fungal infection, either L-AmB (in case of side effects or contraindication related to amphotericin B) or VOR was initiated. VOR was continued as an oral therapy after the infection responded to systemic therapy, and the patient was able to receive oral therapy. Cavitation, air-crescent sign, and halo sign were classified as the major changes indicative of invasive pulmonary aspergillosis (IPA). Nodules and new infiltrates, including consolidation and effusions, were classified as minor changes. ${ }^{8}$ Possible IPA was defined as highly suggestive clinical and radiological findings of infection without histopathological and/or microbiological evidence of infection. Probable IPA was defined as a positive culture for Aspergillus species from a respiratory specimen or two consecutive Aspergillus galactomannan antigen tests with an index of $\geq 0.5$ with clinical and radiological findings suggestive of IPA. Proven IPA was defined as histopathological evidence of tissue invasion and damage by Aspergillus species with clinical and radiological findings. ${ }^{9}$

First-line antifungal therapy was defined as the initial antifungal treatment. Salvage therapy was defined as antifungal therapy, which was administered after antifungal treatment that failed to achieve clinical and microbiological responses. Posaconazole (POS) was being used for primary antifungal prophylaxis and being administered orally as being a dose of $200 \mathrm{mg}$ three times per day with food and beverage, which increased the absorption of POS. This protocol was being used while the neutrophil count remained to be below $1 \times 10^{9} / \mathrm{L}$ subsequent to chemotherapy and continued until the count recovered to $1 \times 10^{9} / \mathrm{L}$. Secondary antifungal prophylaxis was being administered to patients diagnosed with IPA clinically or microbiologically that developed subsequent to chemotherapy. This prophylaxis was being administered as oral VOR with a dose of $200 \mathrm{mg}$ twice per day or POS with a dose of 200 mg three times per day while the neutrophil count remained to be below $1 \times 10^{9} / \mathrm{L}$ subsequent to chemotherapy, and being continued until the count recovered to $1 \times 10^{9} / \mathrm{L}$. If the patient 
was unable to receive oral therapy, secondary antifungal prophylaxis was administered intravenously. Antibiotic prophylaxis was not being administered to patients.

The defined daily dose (DDD) is described as the assumed average maintenance dose per day for a drug that is used for its main indication in adults. The DDD should reflect the global dosage, irrespective of the genetic variability of drug metabolism. The basic principle is to assign only one DDD per route of administration within an Anatomical Therapeutic Chemical (ATC) code. A DDD will only be assigned for drugs that already have an ATC code. POS (0.8 g, J02AC04), VOR (0.4 g, J02AC03), CAS (50 mg, J02AX04), and amphotericin B (35 mg, J02AA01) were considered when calculating the costs of antimicrobial drugs, according to the WHO Collaborating Center for Drug Statistics methodology. ${ }^{10}$ The costs of drugs without ATC codes were calculated by taking into an account a daily dose at $3 \mathrm{mg} / \mathrm{kg} / \mathrm{day}$, and $250 \mathrm{mg}$ was defined for L-AmB. ${ }^{4}$ Antifungal drug use density was calculated as the yearly patient daily dose (PDD)/100 patient days. The costs of antimicrobial drugs were calculated by converting the price that was billed to the Republic of Turkey Social Insurance Institution per patient using the United States dollar (\$) exchange rate.

\section{Statistical analysis}

Continuous variables were represented as the mean \pm standard deviation and the range. Percentile values were represented with two decimal values. The overall 30 -day crude mortality rate is the number of deaths within 30 days of neutropenia divided by the number of all patients. The infection-related mortality rate is the number of patients who died of infection during the neutropenic episodes divided by the number of all patients. IFI-related mortality rate is the number of patients who died of IFIs within 30 days of diagnosis divided by the number of all patients. Candidemia-related mortality rate is the number of patients who died of candidemia within 30 days of diagnosis divided by the number of patients with candidemia.

\section{Results}

In the study period, 15 of 141 patients who were treated in the hematology ward and ineligible for the study criteria were excluded. During 282 febrile episodes of 126 patients who were evaluated, 24 ( $8 \%$ ) episodes of fungemia were recorded in $18(14 \%)$ patients, respectively (Table 1$)$. The mean age was $51.73 \pm 14.4$ years (17-82 years), and 60 patients were female. The mean Multinational Association for Supportive Care in Cancer score was 17.18 \pm 8.27 (Table 1). The mean duration of $\mathrm{FN}$ was $29.38 \pm 6.95$ days.
Table I Hematological malignancies of the patients

\begin{tabular}{ll}
\hline Hematological malignancies & $\mathbf{n}(\%)$ \\
\hline Acute myeloblastic leukemia & $73(58)$ \\
Acute lymphocytic leukemia & $22(17)$ \\
Non-Hodgkin lymphoma & $7(5)$ \\
Chronic lymphocytic leukemia & $5(4)$ \\
Multiple myeloma & $5(4)$ \\
Hairy cell leukemia & $4(3)$ \\
Aplastic anemia & $3(2)$ \\
Chronic myeloid leukemia & $2(2)$ \\
Plasma cell leukemia & $2(2)$ \\
Mantle cell lymphoma & $2(2)$ \\
Chronic lymphocytic leukemia with Burkitt's lymphoma & $\mathrm{I}(\mathrm{I})$ \\
Total & $126(100)$ \\
\hline
\end{tabular}

\section{Fungal infections and antifungal therapy}

Systemic antifungal drugs were initiated to 23 patients with 31 culture-proven IFIs, 19 patients with probable IPA in 25 febrile neutropenic episodes, 38 patients with possible IPA in 42 febrile neutropenic episodes, and 30 patients with suspected IFI in 31 febrile neutropenic episodes (Table 2). VOR, CAS, and L-AmB were administered as a first-line therapy to treat 72 febrile neutropenic episodes of 65 patients, 45 episodes of 37 patients, and 34 episodes of 32 patients, respectively. Five patients were treated with CAS due to hepatosplenic candidiasis diagnosed by clinical and radiological findings. Of 13 patients who received primary antifungal prophylaxis, six (43\%) patients did not need systemic antifungal treatment under induction or consolidation chemotherapy, and one patient with colostomy could not tolerate antifungal treatment due to diarrhea. Of 23 patients who received secondary antifungal prophylaxis with either oral VOR or oral POS during 46 episodes, systemic antifungal therapy was not needed in 22 of 38 episodes and five of eight episodes, respectively. Secondary antifungal prophylaxis with CAS was found to be effective in six episodes of three patients who had at least two persistent candidemia attacks. Half of those ten cases (50\%) responded to combination therapy. Combinations of VOR and L-AmB were used in three episodes of three patients. Two of those three patients responded to the treatment. VOR and CAS combinations were used empirically in four episodes of four patients, and none of those patients responded to treatment. L-AmB and CAS combinations were used empirically in four episodes of three patients, two of who responded to treatment in three episodes. Oral VOR and POS therapies achieved clinical responses as the maintenance of antifungal therapy in 16 and 18 patients, respectively, at least 4 weeks after systemic antifungal therapy without toxicity or discontinuation. 


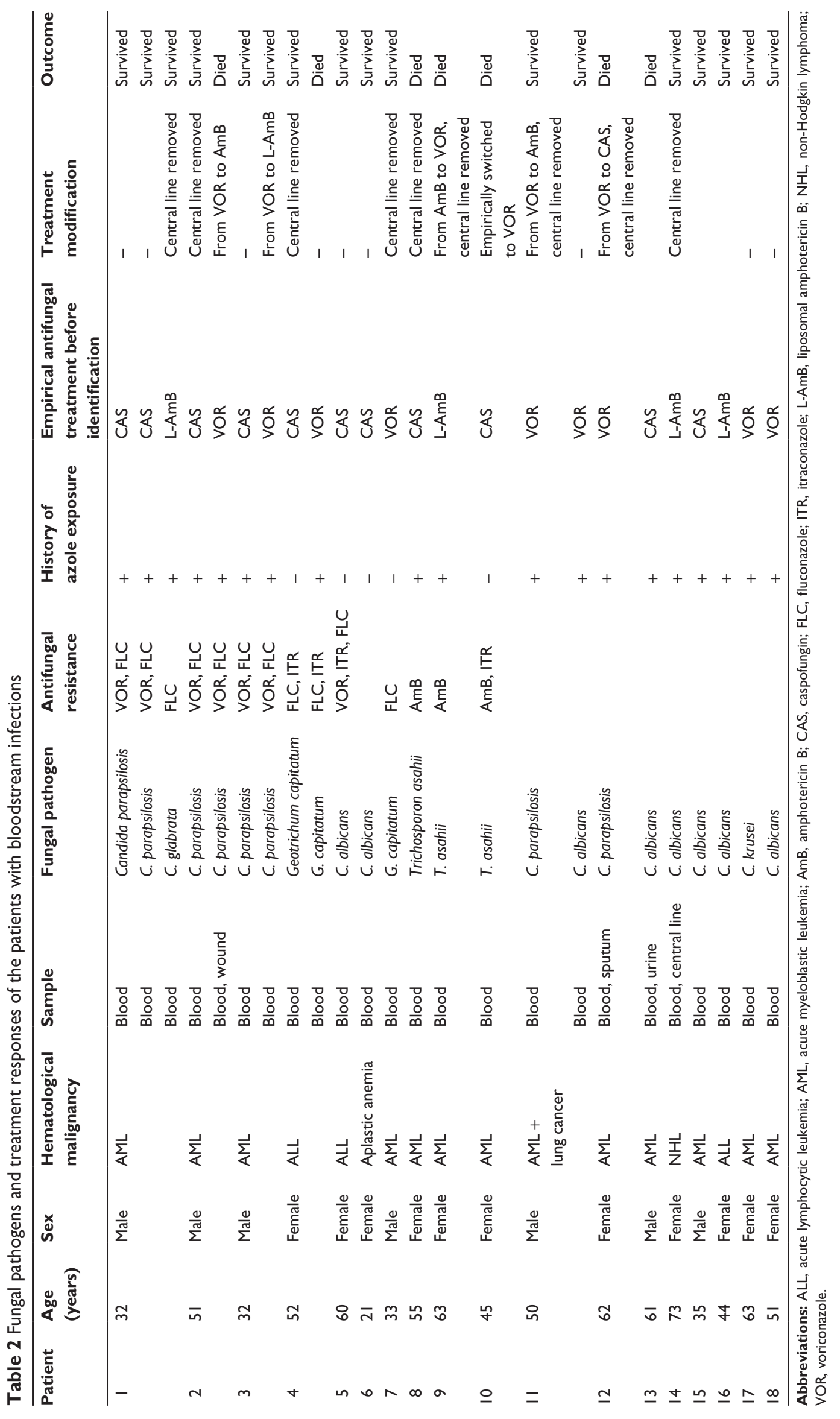




\section{Costs of antimicrobials}

The most commonly used and highest costing antifungal drug was found to be L-AmB with $0.21 \mathrm{PDD} / 100$ patient days, an average cost per month of $\$ 2,9322.98$, and a total cost per year of $\$ 366537.43$; followed by CAS, POS, VOR (intravenous form), VOR (tablet form), respectively (Table 3). Total expenditure of antifungal drugs was calculated as $\$ 1,271,789.08$. The expenditure of antifungal drugs per patient was calculated as $\$ 18,039$. The expenditures of antifungal drugs per febrile neutropenic episode were calculated as $\$ 3,857.85$ for VOR, $\$ 15,783.34$ for CAS, and $\$ 21,561.02$ for L-AmB. The expenditure of POS per patient was $\$ 32,167.39$ for either primary or secondary prophylaxis (Table 3 ).

\section{Mortality}

The overall 30-day crude mortality rates were $31.74 \%(40 / 126)$ among patients diagnosed with acute myelogenous leukemia $(\mathrm{n}=32)$, acute lymphocytic leukemia $(\mathrm{n}=9)$, multiple myeloma $(\mathrm{n}=1)$, chronic myeloid leukemia $(\mathrm{n}=1)$, and non-Hodgkin's lymphoma $(\mathrm{n}=1)$ during the study period. The number of patients who died due to infections was $28(22.22 \%)$. The mortality rates due to IFI and candidemia were $6.3 \%(n=8)$ and $16.66 \%(n=3 / 18)$, respectively.

\section{Discussion}

Antifungal drugs increase the cost of treatment in patients whose FN episodes are secondary to chemotherapy due to hematological malignancies. Especially empirical antifungal treatment increases expenditure, as the diagnosis of IFIs has remained difficult, despite new tests and modalities. IFI was confirmed in $4 \%$ of patients, including only $22 \%-34 \%$ of the neutropenic patients with cancer and received an antifungal drug according to established criteria. ${ }^{11}$ Empirical antifungal therapy is recommended for patients with persistent neutropenic fever after 5-7 days of antibacterial therapy or have clinical or radiological findings related to invasive aspergillosis (IA), such as progressive pulmonary infiltrates on serial CT scanning, to achieve a better outcome, ${ }^{3,8,9}$ because early and appropriate antifungal therapy has been reported to provide better outcomes. ${ }^{12}$ Empirical antifungal therapy is administered even if there is no finding other than fever according to guidelines..$^{1-3,12}$ However, an empirical antifungal approach increases expenditures. Empirical antifungal therapy was initiated in 31 episodes that constituted one-tenth of all episodes in our study. Most of antifungal drugs were administered as preemptive treatment of IPA in our study as well. Prolonged multiple antibiotic use, prolonged and deep neutropenia, and unfavorable conditions in our hematology ward were more likely to result in a higher incidence of IFIs and increased antifungal drug use among our patients..$^{13}$ Laminar flow and the use of a high-efficiency particulate air filter in patient room were reported to prevent reinfection with Aspergillus, but not relapse. ${ }^{14}$ Persistent fever commonly prompts physicians to initiate an empirical antifungal drug. Preemptive antifungal therapy prevents unnecessary antifungal drug use reducing medical costs, but several concerns including the delay of antifungal treatment, which causes poor prognosis, and the lack of established criteria for starting preemptive antifungal agents remain. However, the survival rates obtained with preemptive and empirical antifungal therapies were reported to be similar. ${ }^{12}$ The availability of rapid diagnostic tests and serial CT imaging may lead physicians to choose preemptive antifungal treatment and reduce the expenditures related to antifungal drugs. ${ }^{8,9}$ Moreover, in case the incidence of IA is greater than $4.73 \%$ in the hematology ward, empirical treatment costs less than pre-emptive treatment. If the incidence of IA is $1.64 \%$ or less in a hematology ward, pre-emptive treatment costs less than empirical treatment. ${ }^{15}$ Hematology department should evaluate its treatment strategy based on the incidence of IA. Although conventional amphotericin B is the least expensive antifungal drug and is recommended for empirical antifungal treatment of IPA, this drug could be administered less frequently than other antifungal drugs due to side effects or contraindications. VOR may be used and also

Table 3 Antimicrobials and their costs

\begin{tabular}{|c|c|c|c|c|c|c|c|}
\hline Antifungal drugs & DDD & $\begin{array}{l}\text { PDD/I } 00 \\
\text { patient days }\end{array}$ & $\begin{array}{l}\text { Mean DDD } \\
\text { per month }\end{array}$ & $\begin{array}{l}\text { Range of DDD } \\
\text { per month }\end{array}$ & $\begin{array}{l}\text { Mean cost } \\
\text { per month } \\
\text { (US\$) }\end{array}$ & $\begin{array}{l}\text { Range of costs } \\
\text { per month } \\
\text { (US\$) }\end{array}$ & $\begin{array}{l}\text { Total cost } \\
\text { per year } \\
\text { (US\$) }\end{array}$ \\
\hline Amphotericin B (standard) & $35 \mathrm{mg}$ & 0.08 & 16.04 & $0-34.28$ & 39.06 & $0-880.43$ & 187.12 \\
\hline Voriconazole (tablet form) & $0.4 \mathrm{~g}$ & 0.42 & 162.53 & $6-244$ & $2,796.1 \mathrm{I}$ & $266.46-5,447.22$ & $34,951.42$ \\
\hline Voriconazole (intravenous form) & $0.4 \mathrm{~g}$ & 0.15 & 32.12 & $0-118$ & $14,178.42$ & $0-50,999.94$ & $177,230.33$ \\
\hline Posaconazole & $0.8 \mathrm{~g}$ & 0.09 & 34.28 & $5-70$ & $4,482.97$ & $0-880.43$ & $337,757.63$ \\
\hline Caspofungin & $50 \mathrm{mg}$ & 0.28 & 56.62 & $13.4-154.4$ & $28,4 \mid 0.01$ & $6,954.5-87,220.58$ & $355,125.15$ \\
\hline Liposomal amphotericin B & $250 \mathrm{mg}$ & 0.21 & & & $29,322.98$ & $0-82,261.44$ & $366,537.43$ \\
\hline Total & & & & & & & I,27I,789.08 \\
\hline
\end{tabular}

Abbreviations: DDD, defined daily dose; PDD, patient daily dose. 
cost less than L-AmB for the treatment of possible or probable IPA. Switching to tablet form of VOR may reduce expenditure in the treatment of IPA that prolongs many days when the patient is stable and able to swallow tablet. Although the tablet form of VOR was administered more than its intravenous (IV) form, total expenditure of tablet form was less than IV form in our study. Therefore, the initiation of VOR may be a better choice and reduce expenditures for patients with $\mathrm{FN}$ and persistent fever after antibacterial treatment with $\mathrm{CT}$ findings related to the IPA. However, VOR was reported to be ineffective in $40 \%$ of patients who received targeted, empirical, preemptive prophylactic regimens due to its nonlinear pharmacokinetics. ${ }^{16}$ This drug may also be discontinued due to hallucination accompanied by blurred and colored visual changes or elevated liver enzymes. ${ }^{13,17} \mathrm{CAS}$ and L-Am B, which are recommended for empirical antifungal therapy, increase expenditure more than VOR that is not recommended for empirical antifungal therapy. CAS has been reported to not be more cost-effective than VOR for patients with persistent fever pulmonary findings related to IA during neutropenia. ${ }^{18-20}$

The combination therapy, which is recommended for salvage therapy for IPA, is another factor that increases expenditure in treatment of IFIs. In our study, only ten patients received combination therapy, and half of those patients recovered. Combination therapy should be considered meticulously to prevent increasing expenditures and drug-associated side effects. Antifungal prophylaxis, which was performed as primary and secondary antifungal prophylaxis and increased costs in our study, remains a debated issue. Primary prophylaxis is recommended for patients who undergo a hematopoietic stem cell transplant or intensive remission induction or salvage induction chemotherapy for acute leukemia. ${ }^{1-3}$ POS, which was used for primary and secondary antifungal prophylaxis in our study, is the third highest-priced antifungal drug. Primary antifungal prophylaxis is reported to reduce the incidence of IFIs more than fluconazole (FLC) in patients with acute myelogenous leukemia or myelodysplastic syndrome with similar mortality rates and to be effective in selected patients, taking into account its cost-effectiveness and side-effect. ${ }^{21,22}$ For instance, prophylaxis has been reported to increase azoleresistant fungal infections. However, secondary antifungal prophylaxis has been reported to be effective. ${ }^{13,23}$ Secondary antifungal prophylaxis with VOR may cost less and prevent reactivation of previous aspergillosis. ${ }^{14}$ VOR has not been recommended for primary antifungal prophylaxis yet. Primary antifungal prophylaxis is not recommended in settings that have a relatively high incidence of invasive mold infection and that do not have facilities for early diagnosis and treat- ment. ${ }^{24}$ IFIs were reported to develop in two of 24 patients who had received primary antifungal prophylaxis with POS and in one of 24 patients who had received primary antifungal prophylaxis with oral FLC. ${ }^{25}$ Therefore, FLC can be administered also as primary antifungal prophylaxis to reduce costs in health care settings with limited resources. CAS has been reported to be ineffective for primary antifungal prophylaxis, but this drug can be considered for secondary antifungal prophylaxis for patients colonized with FLC-resistant Candida species, and those with a history of invasive Candida infection. ${ }^{18,26}$

It was reported that use of VOR is more cost effective than the use of AmB (conventional and liposomal) in a multicenter study conducted in five hospitals where the bed capacity varied from 1,000 to 1,700 beds. Average AmB consumption decreased from 5.8 to $2.4 \mathrm{PDD} / 100$ patient days for conventional AmB and from 1.6 to 0.6 for L-AmB, respectively. VOR use, which $80 \%$ of all doses were administered in the hematology-oncology ward by the oral route, increased from zero to a use density of 10.3 PDD/100 patient days in $2003 .{ }^{4}$ In the previous studies investigating pharmacoeconomic status of antifungal drugs administered in the empirical treatment of IFIs in Turkey, CAS was reported to cost less than L-AmB and VOR for expenditure. ${ }^{27-29}$ The costs were reported as approximately US\$1,414, 16,328, and 1,415 per treated patient, successfully treated patient and surviving patient, respectively. ${ }^{27}$ In addition, VOR has been reported to be more favorable than L-AmB ${ }^{28}$ However, decision analytic modeling was used in those studies in contrast to our study that expenditures of antifungal drugs were calculated retrospectively. Expenditures per patient calculated in our study were similar to those reported in their studies. L-AmB was the most expensive antifungal drug in our study and in other studies as well. ${ }^{16,30} \mathrm{~L}$-AmB could be initiated in patients who have IA and develop side effects under VOR therapy as occurred in our study. Antifungal drug selection should be tailored to patient and health care setting's conditions.

\section{Conclusion}

Improving conditions in the patient's room, choosing preemptive antifungal treatment instead of empirical antifungal treatment, switching to tablet form of VOR after initiation of its intravenous form, administration of VOR secondary prophylaxis for invasive aspergillosis instead of primary prophylaxis with POS, and less preference of L-AmB as being an alternative to other antifungal drugs may reduce expenditures related to use of antifungal drugs in the treatment of invasive fungal infections during febrile neutropenic episodes of patients with hematological malignancies. 


\section{Disclosure}

The author reports no conflicts of interest in this work.

\section{References}

1. Hughes WT, Armstrong D, Bodey GP, et al. 2002 Guidelines for the use of antimicrobial agents in neutropenic patients with cancer. Clin Infect Dis. 2002;34:730-751.

2. Freifeld AG, Bow EJ, Sepkowitz KA, et al. Infectious Diseases Society of America. Clinical practice guideline for the use of antimicrobial agents in neutropenic patients with cancer: 2010 update by the Infectious Diseases Society of America. Clin Infect Dis. 2011;52:56-93.

3. Maertens J, Marchetti O, Herbrecht R, et al. European guidelines for antifungal management in leukemia and hematopoietic stem cell transplant recipients: summary of the ECIL 3-2009 update. Bone Marrow Transplant. 2011;46:709-718.

4. de With K, Steib-Bauert M, Knoth H, et al. Hospital use of systemic antifungal drugs. BMC Clin Pharmacol. 2005;5:1-6.

5. Stam WB, Aversa F, Kumar RN, Jansen JP. Economic evaluation of caspofungin versus liposomal amphotericin B for empiric antifungal treatment in patients with neutropenic fever in Italy. Value Health. 2008;11:830-841.

6. Naik S, Lundberg J, Kumar R, Sjolin J, Jansen JP. Economic evaluation of caspofungin versus liposomal amphotericin $\mathrm{B}$ for empirical antifungal therapy in patients with persistent fever and neutropenia in Sweden. Scand J Infect Dis. 2011;43:504-514.

7. Kaskel P, Tuschy S, Wagner A, et al. Economic evaluation of caspofungin vs liposomal amphotericin B for empirical therapy of suspected systemic fungal infection in the German hospital setting. Ann Hematol. 2008;87:311-319.

8. Caillot D, Couaillier JF, Bernard A, et al. Increasing volume and changing characteristics of invasive pulmonary aspergillosis on sequential thoracic computerised tomography scans in patients with neutropenia. J Clin Oncol. 2001;19:253-259.

9. Ascioglu S, Rex HB, De Pauw B, et al. Defining opportunistic invasive fungal infections in immunocompromised patients with cancer and hematopoietic stem cell transplants: an international consensus. Clin Infect Dis. 2002;34:7-14.

10. WHO Collaborating Centre for Drug Statistics and Methodology. Guidelines for ATC Classification and DDD Assignment. Oslo, Norway: WHO, 2003. Available from: http://www.whocc.no/ddd/definition_and_general_considera. Accessed December 17, 2009.

11. Walsh TJ, Finberg RW, Arndt C, et al. Liposomal amphotericin B for empirical therapy in patients with persistent fever and neutropenia. National Institute of Allergy and Infectious Diseases Mycoses Study Group. N Engl J Med. 1999;340:764-771.

12. Cordonnier C, Pautas C, Maury S, et al. Empirical versus preemptive antifungal therapy for high-risk, febrile, neutropenic patients: a randomized, controlled trial. Clin Infect Dis. 2009;48:1042-1051.

13. Gedik H, Şimşek F, Yıldırmak MT, et al. Primary or secondary antifungal prophylaxis in patients with hematological maligancies: efficacy and damage. Ther Clin Risk Manag. 2014;10:305-312.

14. Sipsas NV, Kontoyiannis DP. Clinical issues regarding relapsing aspergillosis and the efficacy of secondary antifungal prophylaxis in patients with hematological malignancies. Clin Infect Dis. 2006;42: 1584-1591.
15. Kimura SI, Murata T, Akahoshi Y, et al. Economic evaluation of a preemptive treatment strategy for invasive fungal infection in neutropenic patients with hematological diseases. Eur J Clin Microbiol Infect Dis. 2015;34:951-961.

16. Nivoix Y, Launoy A, Lutun P, et al. Adherence to recommendations for the use of antifungal agents in a tertiary care hospital. J Antimicrob Chemother. 2012;67:2506-2513.

17. Mikulska M, Novelli A, Aversa F, et al. Voriconazole in clinical practice. J Chemother. 2012;24(6):311-327.

18. Gedik H, Şimşek F, Yıldırmak MT, et al. Novel antifungal drugs against fungal pathogens: do they provide promising results for treatment? Indian J Hematol Blood Transfus. 2015;31:196-205.

19. Domínguez-Gil A, Martín I, García Vargas M, Del Castillo A, Díaz S, Sánchez C. Economic evaluation of voriconazole versus caspofungin for the treatment of invasive aspergillosis in Spain. Clin Drug Investig. 2007;27(3):197-205.

20. Kaskel P, Tuschy S, Wagner A, et al. Economic evaluation of caspofungin vs liposomal amphotericin B for empirical therapy of suspected systemic fungal infection in the German hospital setting. Ann Hematol. 2008;87:311-319.

21. Kung HC, Johnson MD, Drew RH, Saha-Chaudhuri P, Perfect JR. Clinical effectiveness of posaconazole versus fluconazole as antifungal prophylaxis in hematology-oncology patients: a retrospective cohort study. Cancer Med. 2014;3:667-673.

22. Girmenia C, Frustaci AM, Gentile G, et al. Posaconazole prophylaxis during front-line chemotherapy of acute myeloid leukemia: a singlecenter, real-life experience. Haematologica. 2012;97(4):560-567.

23. Liu M, Li Y, Zhang Y, et al. Secondary antifungal prophylaxis in hematological malignancy patients with previous invasive fungal disease: a retrospective analysis. PLoS One. 2014;9:e115461.

24. De PB, Donnelly JP. Prophylaxis and aspergillosis - has the principle been proven? N Engl J Med. 2007;356:409-411.

25. Gomes MZ, Mulanovich VE, Jiang Y, Lewis RE, Kontoyiannis DP Incidence density of invasive fungal infections during primary antifungal prophylaxis in newly diagnosed acute myeloid leukemia patients in a tertiary cancer center, 2009 to 2011. Antimicrob Agents Chemother. 2014;58(2):865-873.

26. Hughes WT, Armstrong D, Bodey GP, et al. 2002 Guidelines for the use of antimicrobial agents in neutropenic patients with cancer. Clin Infect Dis. 2002;34:730-751.

27. Turner SJ, Senol E, Kara A, Al-Badriyeh D, Dinleyici EC, Kong DCM. Cost effectiveness of caspofungin vs voriconazole for empiric therapy in Turkey. Mycoses. 2014;57(8):489-496.

28. Turner SJ, Senol E, Kara A, Al-Badriyeh D, Dinleyici EC, Kong DCM. Pharmacoeconomic evaluation of voriconazole vs liposomal amphotericin B in empiric treatment of invasive fungal infections in Turkey. BMC Infect Dis. 2013;13:560.

29. Turner SJ, Senol E, Kara A, Al-Badriyeh D, Kong DCM, Dinleyici EC. Pharmacoeconomic evaluation of caspofungin versus liposomal amphotericin B in empirical treatment of invasive fungal infections in Turkey. Int J Antimicrob Agents. 2013;42(3):276-280.

30. Parr MD, Waite WW, Hansen LA, McDaniel PA. Cost comparison of seven antibiotic combinations as empiric therapy in a simulated febrile neutropenic patient. Am J Hosp Pharm. 1985;42(11):2484-2488.
ClinicoEconomics and Outcomes Research

\section{Publish your work in this journal}

ClinicoEconomics and Outcomes Research is an international, peerreviewed open-access journal focusing on Health Technology Assessment, Pharmacoeconomics and Outcomes Research in the areas of diagnosis, medical devices, and clinical, surgical and pharmacological intervention. The economic impact of health policy and health systems

\section{Dovepress}

organization also constitute important areas of coverage. The manuscript management system is completely online and includes a very quick and fair peer-review system, which is all easy to use. Visit http://www.dovepress.com/testimonials.php to read real quotes from published authors. 